\title{
Staining Interactions of Bixa Orellana (Annatto) Seed Extracts with Tissues of Wistar Rats
}

\section{Okorie Nnaemeka1, Avwioro 0G², Fasogbon Samuel A ${ }^{3 *}$, Abubakar Usman $^{2}$, Adeluwoye Adekunle $\mathrm{O}^{4}$, Agada Uchechukwu $\mathrm{N}^{5}$, Okoro Godwin $0^{1}$ \\ ${ }^{1}$ Department of Medical Laboratory Science, Faculty of Health Sciences, Ebonyi State} University, Nigeria

${ }^{2}$ Department of Histopathology, School of Medical Laboratory Science, Usmanu Danfodiyo University, Nigeria ${ }^{3}$ Public health in-vitro Diagnostics Control Laboratory, Medical Laboratory Science Council of Nigeria, Nigeria ${ }^{4}$ Department of Medical Laboratory Science, Lead City University, Nigeria ${ }^{5}$ Histopathology Unit, Medical Laboratory Services, Alex Ekwueme Federal University Teaching Hospital, Nigeria

*Corresponding author: Fasogbon, Samuel Ayobami, Public health in-vitro Diagnostics Control Laboratory, Medical Laboratory Science Council of Nigeria (MLSCN), Lagos, Nigeria, Tel: +2348032229904; Email: samfash4best@gmail.com

\section{Abstract}

Introduction: Bixa orellana is an ancestral multi use plant popularly known as Achiote or lipstick tree in view of its reddish - orange dye on its seeds, Central and South American populations used these seeds to color their bodies and lips, B. orellana is the only species of Bixaceae family.

Aim: The aim of this study was to stain the organs such as skin, liver, kidney, brain, intestines, and bone marrow of a wistar rats with solutions of Bixa orellana (Annatto) seed extracts.

Methods: The study design was experimental research, the seeds of Bixa orellana were extracted in distilled water, absolute ethanol and acetone, using maceration methods and the fractions of the extracts were determined using column chromatography while preliminary, the solutions of the extracts were used to stain tissues listed above at varying $\mathrm{pH}$ to establish staining interactions of the crude extracts and fractions eluted from the column chromatography.

Results: The most significant staining interaction were observed in ethanol solution of bixa extract, followed by acetone and distilled water and staining were also observed at column fraction(9-26), with background yellowish appearance in all the staining solutions which was contrast to controls (haematoxylin and eosin) stained sections and no staining reaction was observed in blood smear of wistar rat. The staining interactions of great quality was observed at pH 5.0 and 7.5, despite its staining ability it was neither superior to eosin nor a substitute to giemsa stain due to the yellowish background appearance and its complete inactivity on blood smear. 
Conclusion: Bixa orellana dye extracts can be grouped under compound dyes or neutral dyes due to its interaction at both pH 5.0 and 7.5 and cannot be used as substitute for Giemsa stain.

Keywords: Blood Smear; Bixa Orellana; Eosin; Giemsa Stain; Organs.

\section{Introduction}

Bixa orellana plant popularly known as Achiote or lipstick tree got its name in view of its reddish - orange dye on its seeds, the Ibos in South Eastern Nigeria discovered it during Nigeria-Biafra war and use it then for cosmetic reasons and today it has formed part of ornamental shrubs in the region meanwhile in the course of this research it was transported and planted to the Northern Nigeria (Usmanu Danfodiyo Teaching Hospital Sokoto). Central and South American populations used these seeds to color their bodies and lips. B. orellana is the only species of Bixaceae family [1].

The species name of this plant is named after the Spanish scientist conquistador, Franscisco de orellana. The dye obtained from a thin, highly coloured resinous coating of triangular seeds present in brown or crimson capsular fruit as shown in figure 1 is popularly called "annatto" colourant (E-160B). Some of the common synonyms are CI Natural Orange 4; CI 75120, Achiote, Annotta, Annatta, Annatto, Annotta, Bija, Rocou, Roucou, Roucouyer, Roucoyer, Orlean, Orleanstraugh, Terre orellana, Beni-No-Ki, Urucu, Urucum, L. Annatto is known for its lack of toxicity, its high tinctorial value and its high range of colour- comprising of red, orange and yellow hues [2]. B. orellana is a tropical shrub, native to South American countries and its effective cultivation is reported in many parts of the world [3].

Bixa orellana (annatto) plant is popularly known as Achiote or lipstick tree due to its reddish orange dye on its seeds. The dye is highly concentrated in the thin coloured resinous coating triangular seeds present in the brown or crimson capsular fruits [4]. Annatto has been applied in the production of foods and drugs as a colourant, according to Nguyen PM [4] the oil soluble annatto colour is used in dairy and fats based products like butter, margarine, cheese, bake, pharmacy and also leather and cosmetics. Available literatures provide information on chemical structure of bixa orellana, its extraction, formulation, processing, pharmacological and its toxicology [5]. According to Nguyen PM [4] many technical factors have been identified to influence annatto extraction such as concentration of solvent, ratio of solute to solvent, extraction time, and extraction temperature. However satisfactory results of annatto were obtained by the use of UV-xis spectroscope analysis and Fourier transform infrared spectroscopy analysis (FTIR). FTIR has also helped to identify the chemical structure of the extract corresponding to the carotenoid compounds [5]. Considering the restrictions placed on the use of synthetic dyes by world health organization, interest in natural dyes is increasing, however, annatto seed pigments-bixin and norbixin are among those most used in the food, pharmacological and cosmetic industries due to the intensity of their colours, their greater stability and wide variety of colours is an additional advantage of the annatto carotenoids over other carotenoids such as those of carrot and beetroot. In food production, extraction with $\mathrm{CO}_{2}$ has shown satisfactory advantage since it is inert gas under normal condition it is easy to separate from the solute hence obtaining a pure final product with organic solvent residues [6].

The aim of this study is to determine the staining interaction of bixa orellana (annatto) seed extracts with tissues of wistar rat, as a counter stain to haematoxylin and a substitute to giemsa stain respectively.

\section{Methodology}

\section{Study Location}

The study was carried out in histopathology department Faculty of Medical Laboratory science, Department of Pharmacognosy and Ethno Pharmacy Faculty of Pharmaceutical science Usmanu Danfodiyo University, Sokoto and in collaboration with Usmanu Danfodiyo University Teaching Hospital, Sokoto.

\section{Plant Identification}

The plant taxonomic identification and assigning of specimen Voucher Number was carried out by Malam Abdulazeez Salihu from the Botany unit, Department of Biological Sciences Usmanu Danfodiyo University Sokoto, and a voucher specimen (UDUS/ANS/0157) was prepared and deposited in the herbarium of the same department 


\section{Study Design}

This study was experimental research designed and the seeds of Bixa orellana were extracted in distilled water, absolute ethanol and acetone, using maceration methods and the fractions of the extracts were determined using column chromatography while preliminary, the solutions of the extracts were used to stain tissues listed above at varying $\mathrm{pH}$ to establish staining interactions of the crude extracts and fractions eluted from the column chromatography.

\section{Slide Preparation}

Eighteen (18) sets of serial sections labeled A to $S$ were made from a block of processed skin, liver, brain, kidney and intestine tissue of an adult wistar rat and its blood smeared, stained with Harris haematoxylin and counter stained with different preparations at varying $\mathrm{pH}$ of bixa orellana extract and compared with haematoxylin and eosin technique and giemsa respectively.

\section{Laboratory Analysis}

The extract of bixa orellana seeds was subjected to Thin Layer Column Chromatography (TLC) to obtain the fractions and eluents used for this work. The sections of all tissues used were stained with $\mathrm{H}$ and $\mathrm{E}$ stain as control and various preparations of the extract of bixa orellana seeds were used to stain the sections as the test

\section{Statistical Analysis}

The results obtained from staining reactions of bixa orellana seeds extracts were photomicrographically presented and analysed after photomicrograph sections of all the staining interventions were taken and compared with $\mathrm{H}$ and $\mathrm{E}$ staining technique and different preparations of the bixa orellana seeds extracts at varied $\mathrm{pH}$, same solutions staining interaction of blood smear were also photomicrograph and compared with its control giemsa stain.

\section{Results}

\begin{tabular}{|c|c|c|c|c|c|}
\hline Texture & Weight of powder (g) & Extracting solvents & Weight of extracts & \% yields & Colours \\
\hline & 80 & Acetone & 24.00 & 30.00 & Deep red \\
\hline Dried & 80 & Ethanol & 18.38 & 22.97 & Light orange \\
\hline & 80 & Distilled water & 15.20 & 19.00 & Golden yellow \\
\hline
\end{tabular}

Table 1: Physical Characteristics And Percentage (\%) Yield Of Bixa Orellana Seeds.

\section{Group A: Interaction of Aqueous Extract of Bixa on Liver Tissues}

The above Group A photomicrographs were the liver sections of adult Wistar rat, plate 1 is the controlled sections stained with haematoxylin and eosin dye, demonstrated the central vein of the liver (white arrow) while plate 2 and 3 were the photomicrograph sections of the liver sections stained with Haematoxylin and counter stained with aqueous seeds extracts of bixa orellanna at $\mathrm{pH} 5.0$ and 7.5 respectively, demonstrated the central vein of the liver (white arrow) however, the optical differentiation of the interaction of the aqueous extracts were not better than the Eosin control counter stain due to its poor nuclear cytoplasmic appearance of the tissue and yellowish background deposition.

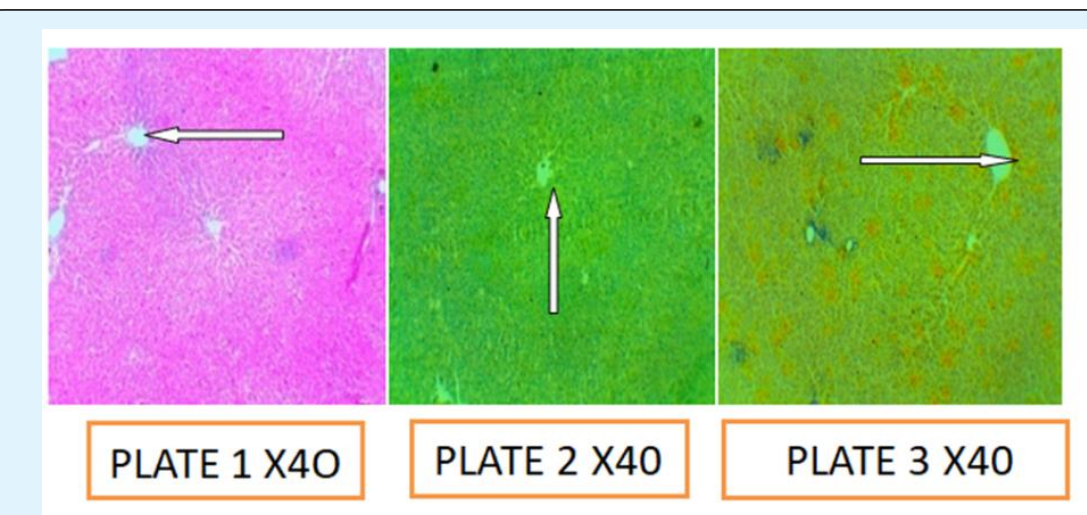

Figure 1: plate1: control; plate 2: $\mathrm{pH}$ 5.0; plate 3: pH: 7.5. 


\section{Group B: Reaction of Ethanolic Extract of Bixa on Liver Tissues}

The above Group B photomicrographs were the liver sections of adult Wistar rat, plate 1 is the controlled sections stained with haematoxylin and eosin dye, the dye demonstrated the central vein of the liver (white arrow) while plate 2 and 3 were the photomicrograph sections of the liver sections stained with Haematoxylin and counter stained with ethanolic seeds extracts of bixa orellanna at pH 5.0 and 7.5 respectively,demonstrated the central vein of the liver (white arrow) however, the optical differentiation of the interaction of the ethanolic extracts were not better than the Eosin control counter stain due to its poor nuclear cytoplasmic appearance of the tissue and yellowish background deposition.

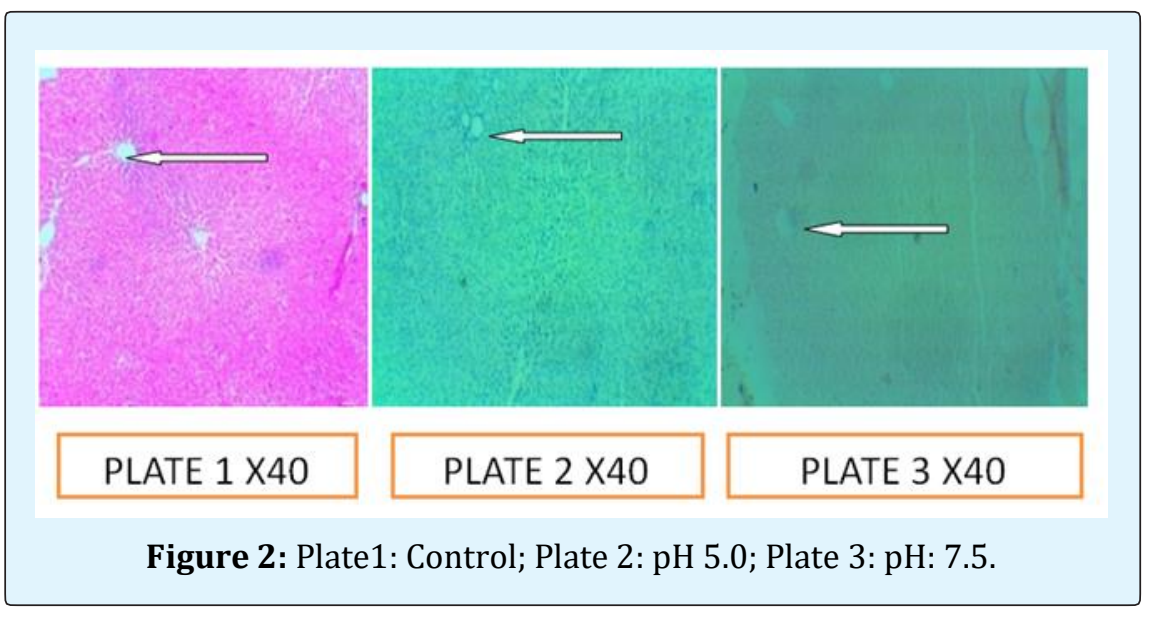

\section{Group C: Reaction of Acetone Extract of Bixa on Liver Tissue}

The above Group C photomicrograph were the liver sections of adult Wistar rat, plate 1 is the controlled sections stained with haematoxylin and eosin dye, the dye demonstrated the central vein of the liver and portal triad while plate 2 and 3 were the photomicrograph sections of the liver sections stained with Haematoxylin and counter stained with acetone seeds extracts of bixa orellanna at pH 5.0 and 7.5 respectively, however, the optical differentiation of the interaction of the acetone extracts were not better than the Eosin control counter stain due to its poor nuclear cytoplasmic appearance of the tissue and yellowish background deposition.

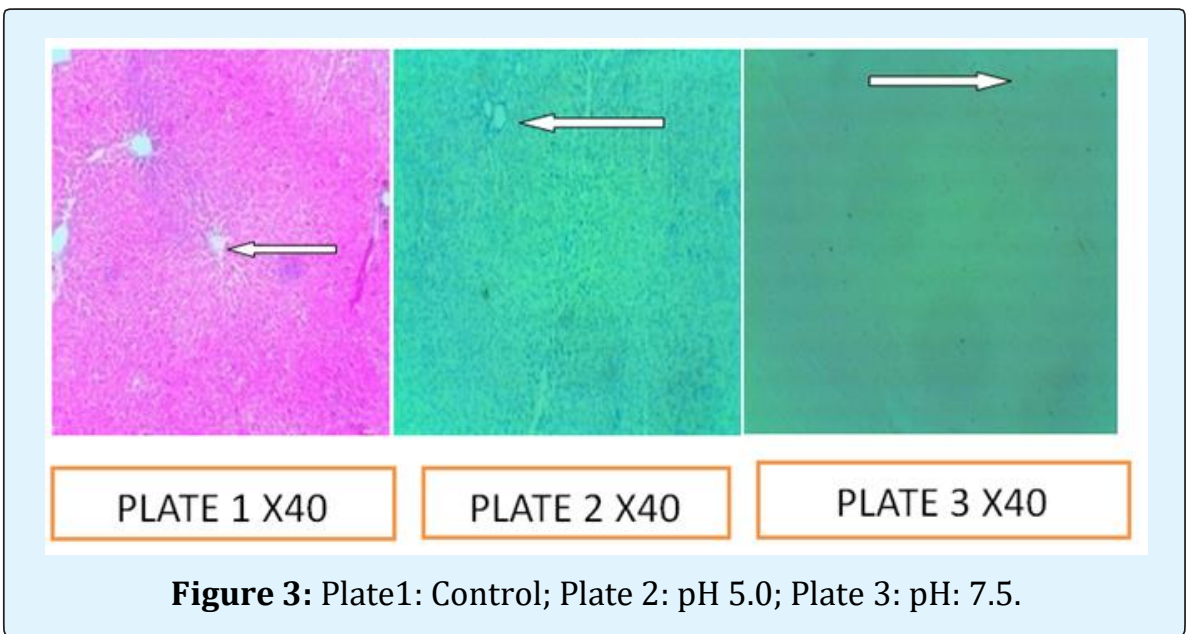

Okorie Nnaemeka, et al. Staining Interactions of Bixa Orellana (Annatto) Seed Extracts with Tissues of Wistar Rats. Ann Adv Biomed Sci 2019, 2(2): 000144. 


\section{Group D: Interaction of Acqueous Extract of Bixa on Brain Tissue}

The above Group D photomicrographs were the brain sections of adult Wistar rat, plate 1 is the controlled sections stained with haematoxylin and eosin dye, demonstrated the white and gray matter while plate 2 and 3 were the photomicrograph sections of the brain sections stained with Haematoxylin and counter stained with aqueous seeds extracts of bixa orellanna at $\mathrm{pH} 5.0$ and 7.5 respectively, also demonstrate gray and white mater, however, the optical differentiation of the interaction of the aqueous extracts were not better than the Eosin control counter stain due to its poor nuclear cytoplasmic appearance of the tissue and yellowish background deposition.

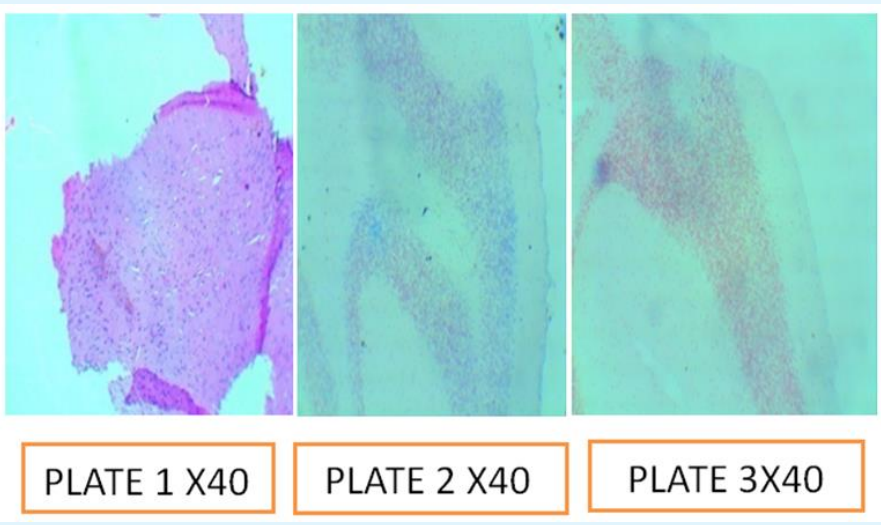

Figure 4: Plate1: Control; Plate 2: pH 5.0; Plate 3: pH: 7.5.

\section{Group E: Interaction of Ethanolic Extract of Bixa Reaction on Brain Tissue}

The above Group E photomicrographs were the brain sections of adult Wistar rat, plate 1 is the controlled sections stained with haematoxylin and eosin dye, demonstrated the white and gray matter while plate 2 and 3 were the photomicrograph sections of the brain sections stained with Haematoxylin and counter stained with ethanolic seeds extracts of bixa orellanna at $\mathrm{pH} 5.0$ and 7.5 respectively, demonstrates the white and gray matter, however, the optical differentiation of the interaction of the ethanolic extracts were not better than the Eosin control counter stain due to its poor nuclear cytoplasmic appearance of the tissue and yellowish background deposition, though preferred when compared aqueous extract.

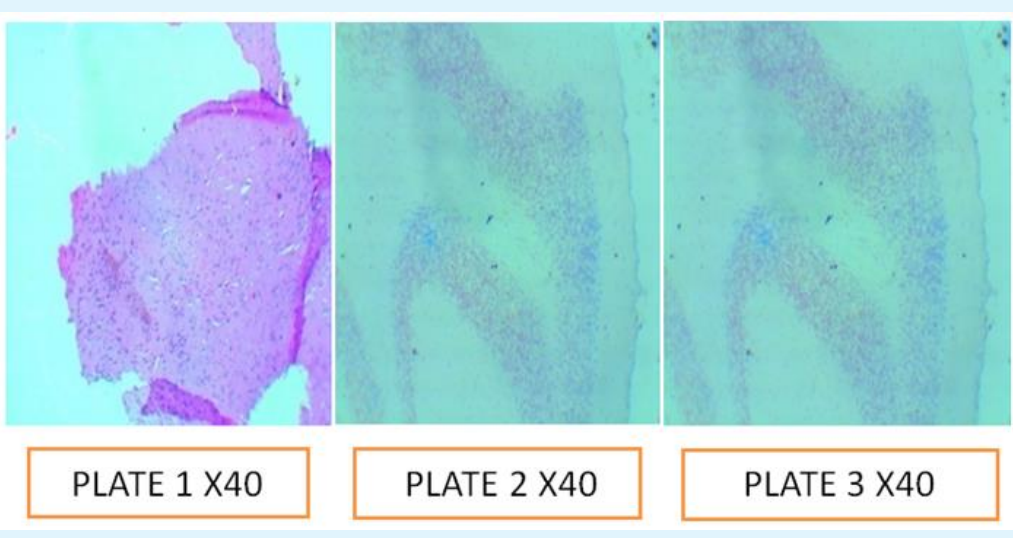

Figure 5: Plate1: Control; Plate 2: pH 5.0; Plate 3: pH: 7.5. 


\section{Group F: Interaction of Acetone Extract of Bixa on Brain Tissue}

The above Group F photomicrographs were the brain sections of adult Wistar rat, plate 1 is the controlled sections stained with haematoxylin and eosin dye, demonstrated the white and gray matter while plate 2 and 3 were the photomicrograph sections of the brain sections stained with Haematoxylin and counter stained with acetone seeds extracts of bixa orellanna at $\mathrm{pH} 5.0$ and 7.5 respectivelydemonstrated the white and gray matter, however, the optical differentiation of the interaction of the acetone extracts were not better than the Eosin control counter stain due to its poor nuclear cytoplasmic appearance of the tissue and yellowish background deposition.

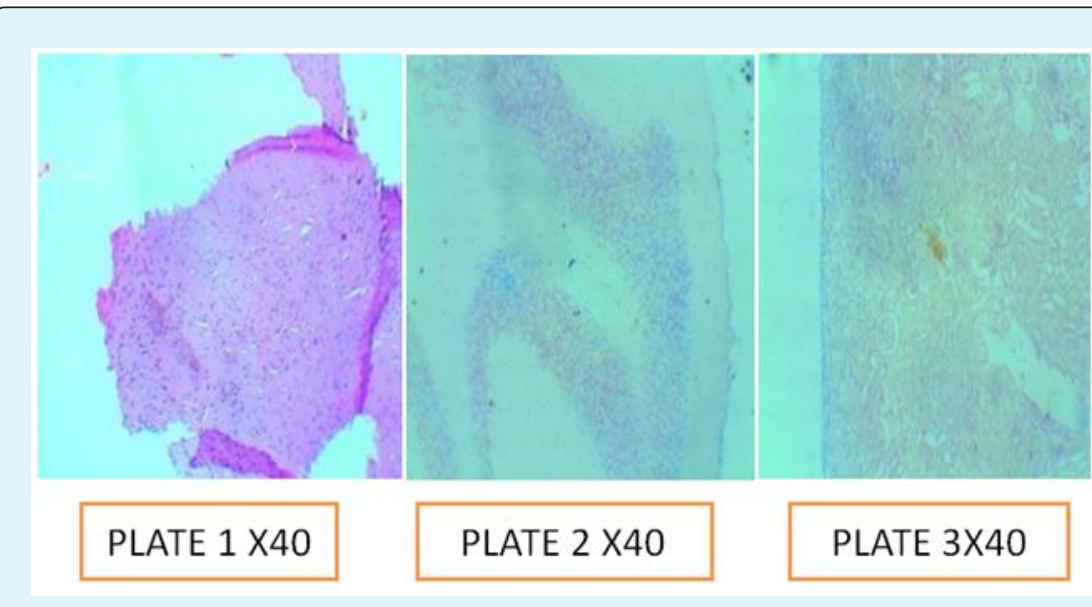

Figure 6: Plate1: Control; Plate 2: pH 5.0; Plate 3: pH 7.5.

\section{Group G: Interaction of Acqueous Extract of Bixa on Intestine Tissue}

The above Group $G$ photomicrographs were the intestine sections of adult Wistar rat, plate 1 is the controlled sections stained with haematoxylin and eosin dye, demonstrated the layers and gastric pit while plate 2 and 3 were the photomicrograph sections of the intestine sections stained with Haematoxylin and counter stained with aqueous seeds extracts of bixa orellanna at $\mathrm{pH} 5.0$ and 7.5 respectively, however, the optical differentiation of the interaction of the aqueous extracts were not better than the Eosin control counter stain due to its poor nuclear cytoplasmic appearance of the tissue and yellowish background deposition.

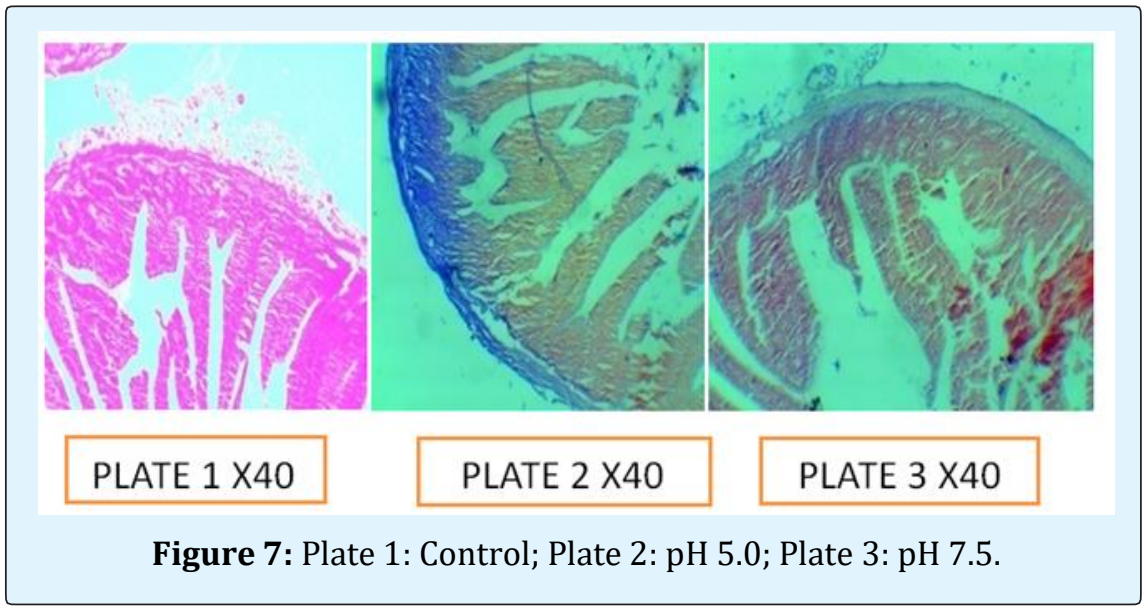

Okorie Nnaemeka, et al. Staining Interactions of Bixa Orellana (Annatto) Seed Extracts with Tissues of Wistar Rats. Ann Adv Biomed Sci 2019, 2(2): 000144. 


\subsection{Group H: Interaction of Ethanolic Extract of Bixa on Intestine Tissue}

The above Group $\mathrm{H}$ photomicrographs were the intestine sections of adult Wistar rat, plate 1 is the controlled sections stained with haematoxylin and eosin dye, the dye demonstrated the layers and gastric pits while plate 2 and 3 were the photomicrograph sections of the intestine sections stained with Haematoxylin and counter stained with ethanolic seeds extracts of bixa orellanna at $\mathrm{pH} 5.0$ and 7.5 respectively, however, the optical differentiation of the interaction of the ethanolic extracts were not better than the Eosin control counter stain due to its poor cytoplasmic appearance of the tissue and yellowish background deposition.

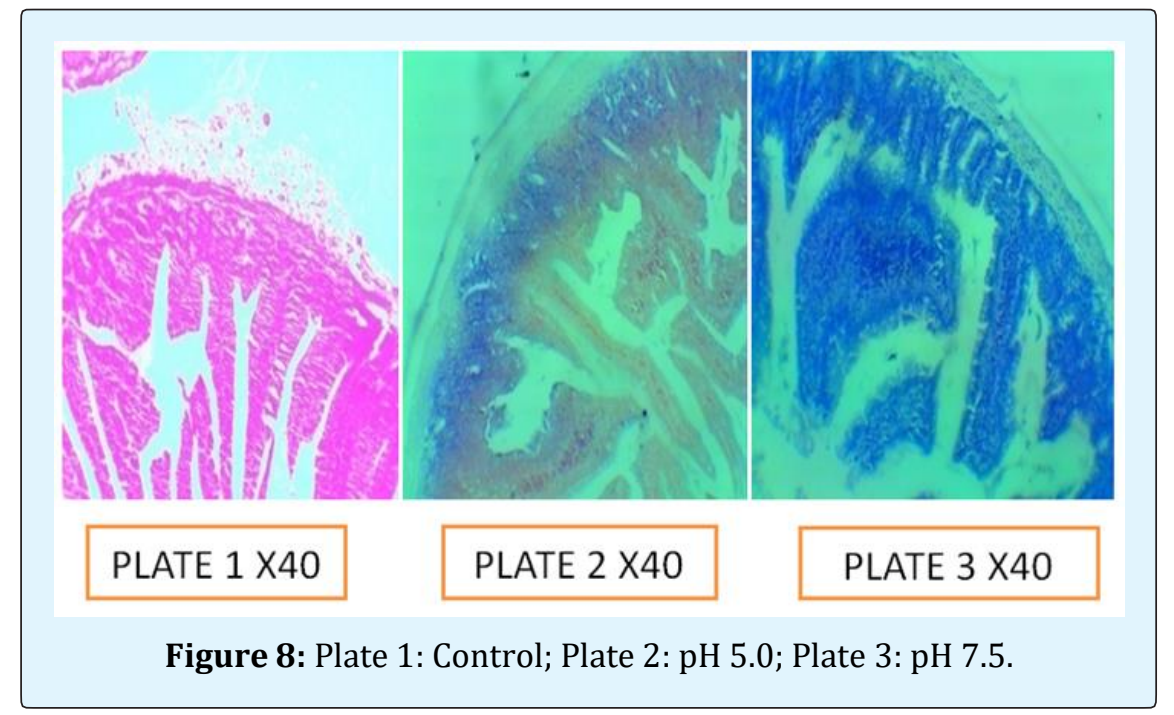

\section{Group I: Interaction of Acetone Extract of Bixa Reaction on Intestine Tissues}

The above Group I photomicrographs were the intestine sections of adult Wistar rat, plate 1 is the controlled sections stained with haematoxylin and eosin dye, the dye demonstrated the goblet cells and gastric pit while plate 2 and 3 were the photomicrograph sections of the intestine sections stained with Haematoxylin and counter stained with acetone seeds extracts of bixa orellanna at $\mathrm{pH} 5.0$ and 7.5 respectively, however, the optical differentiation of the interaction of the acetone extracts were not better than the Eosin control counter stain due to its poor nuclear cytoplasmic appearance of the tissue and yellowish background deposition.

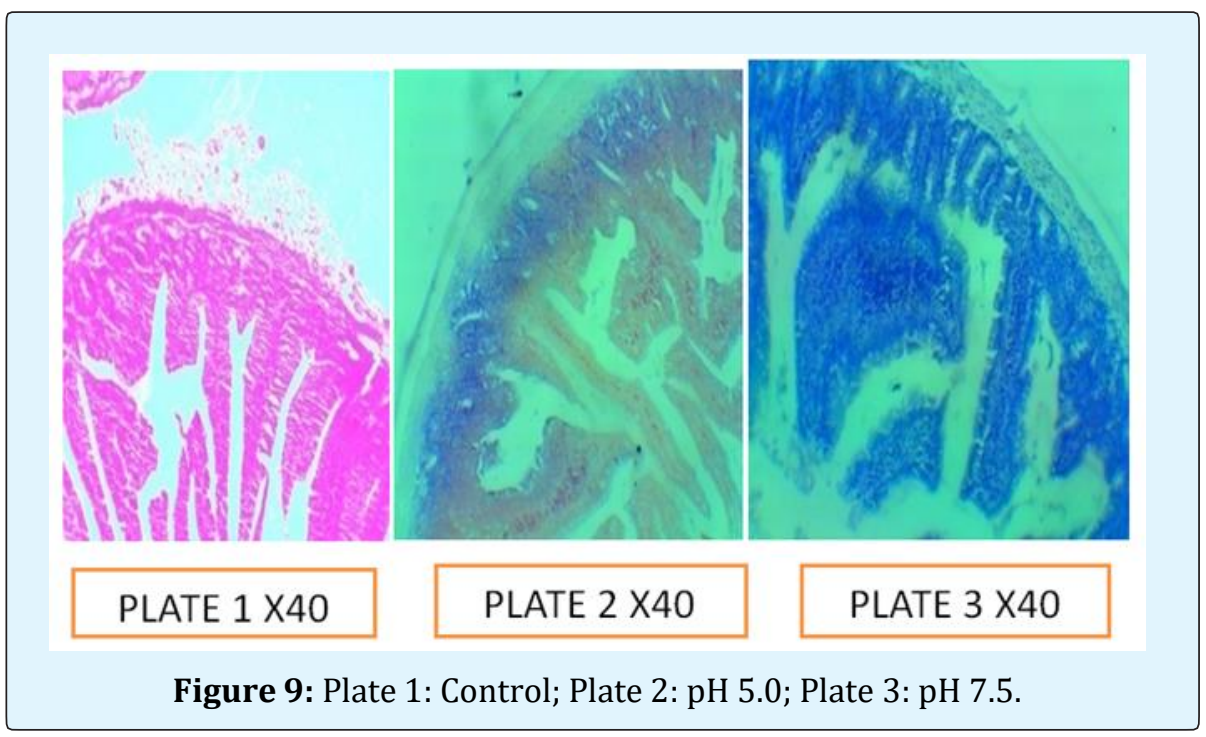

Okorie Nnaemeka, et al. Staining Interactions of Bixa Orellana (Annatto) Seed Extracts with Tissues of Wistar Rats. Ann Adv Biomed Sci 2019, 2(2): 000144. 


\section{Group J: Interaction with Acqueous Extract of Bixa on Skin Tissue}

The above Group J photomicrograph were the skin sections of adult Wistar rat, plate 1 is the controlled sections stained with haematoxylin and eosin dye, the dye demonstrated epidermal papillae of the skin and glandswhile plate 2 and 3 were the photomicrograph sections of the skin sections stained with Haematoxylin and counter stained with aqueous seeds extracts of bixa orellanna at $\mathrm{pH} 5.0$ and 7.5 respectively, it also demonstrated the skin epidermis but not superior to eosin.

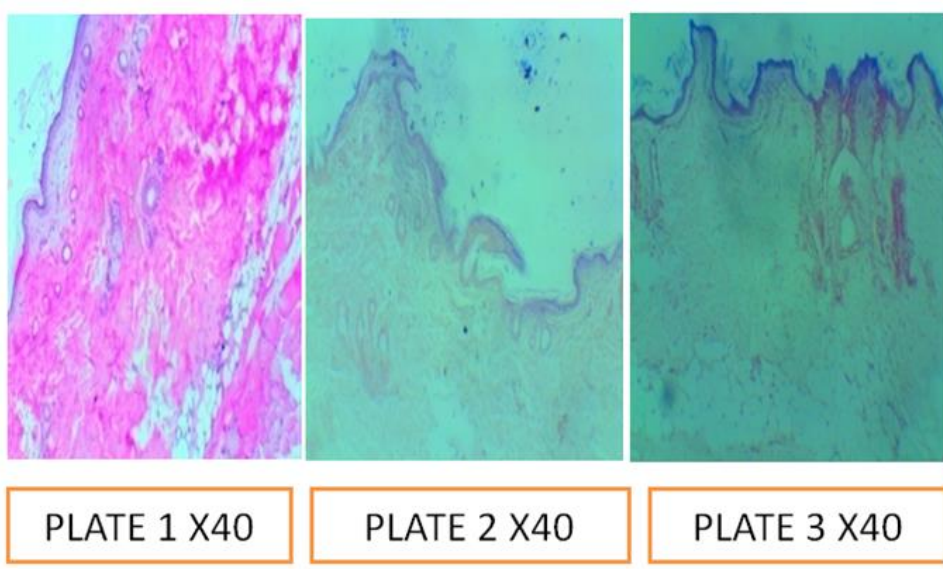

Figure 10: Plate 1: Control; Plate 2: pH 5.0; Plate 3: pH 7.5.

\section{Group K: Reaction of Ethanolic Extract of Bixa on Skin Tissue}

The above Group $\mathrm{K}$ photomicrographs were the skin sections of adult Wistar rat, plate 1 is the controlled sections stained with haematoxylin and eosin dye, the dye demonstrated epidermal papilae of the skin glandswhile plate 2 and 3 were the photomicrograph sections of the skin sections stained with Haematoxylin and counter stained with ethanolic seeds extracts of bixa orellanna at pH 5.0 and 7.5 respectively, it also demonstrated the skin epidermis but not superior to eosin.

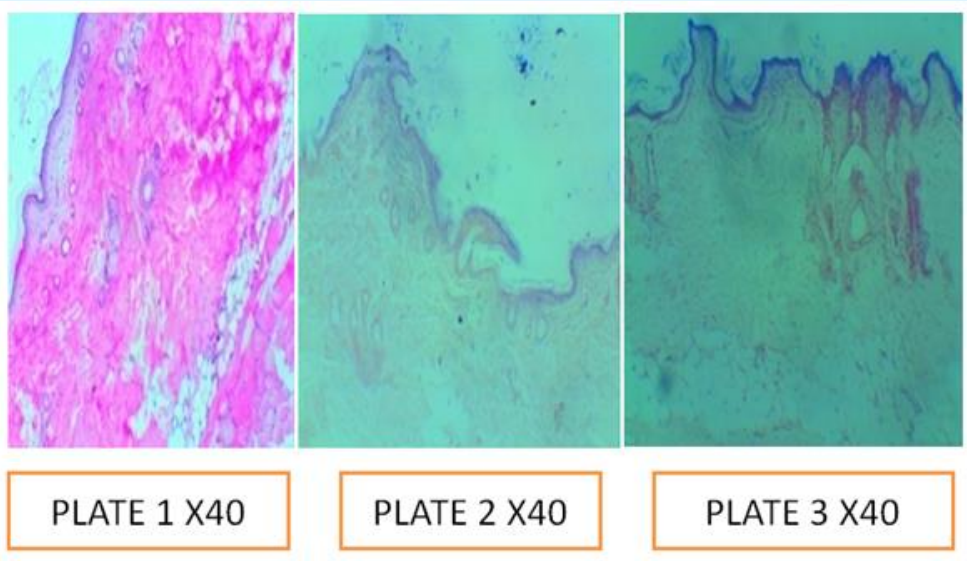

Figure 11: Plate1:Control; Plate 2: $\mathrm{pH}$ 5.0; Plate 3: pH 7.5. 


\section{Group M: Reaction of Acetone Extract of Bixa on Skin Tissue}

The above Group M photomicrographs were the skin sections of adult Wistar rat, plate 1 is the controlled sections stained with haematoxylin and eosin dye, the dye demonstrated epidermal papillae of the skin glands while plate 2 and 3 were the photomicrograph sections of the skin sections stained with Haematoxylin and counter stained with acetone seeds extracts of bixa orellanna at pH 5.0 and 7.5 respectively, it also demonstrated the skin epidermis but not superior to eosin.

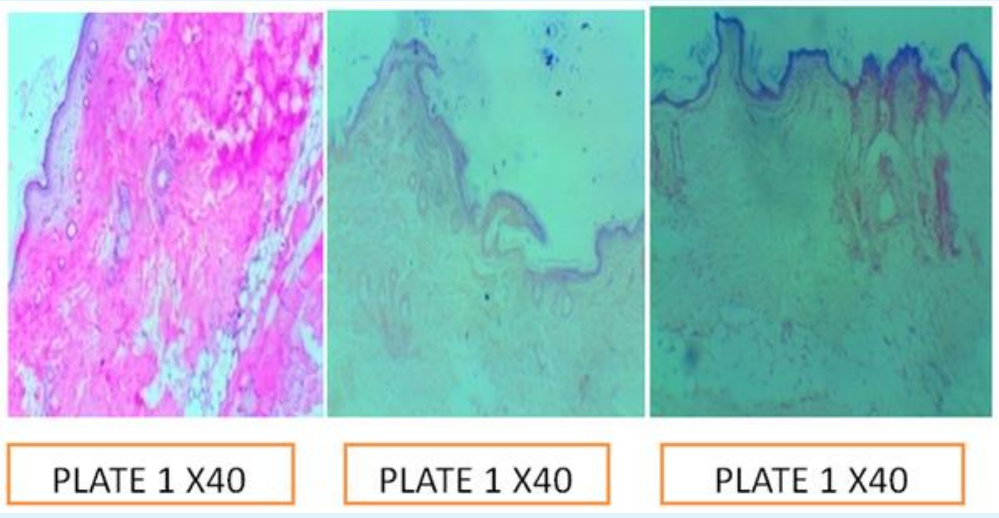

Figure 12: Plate 1: Control; Plate 2: pH 5.0; Plate 3: pH 7.5.

\section{Group N: Interaction of Acqueous Extract of Bixa on Kidney Tissues}

The above Group $\mathrm{N}$ photomicrographs were the kidney sections of adult Wistar rat, plate 1 is the controlled sections stained with haematoxylin and eosin dye, demonstrated glomerular capsule and proximal convoluted tubules while plate 2 and 3 were the photomicrograph sections of the kidney sections stained with Haematoxylin and counter stained with acqueous seeds extracts of bixa orellanna at pH 5.0 and 7.5 respectively, it showed poor optical contrast of the kidney sections when compared with eosin.

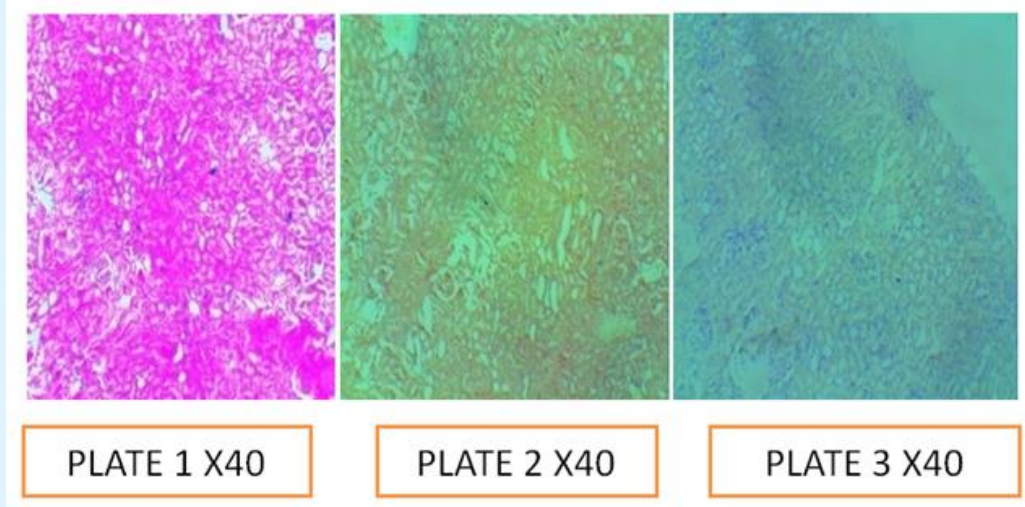

Figure 13: Plate 1: Control; Plate 2: pH 5.0; Plate 3: pH 7.5.

\section{Group 0: Interaction of Ethanolic Extract of Bixa on Kidney Tissue}

The above Group 0 photomicrographs were the kidney sections of adult Wistar rat, plate 1 is the controlled sections stained with haematoxylin and eosin dye, demonstrated glomerular capsule and podocytes while plate 2 and 3 were the photomicrograph sections of the kidney sections stained with Haematoxylin and 
counter stained with ethanolic seeds extracts of bixa orellanna at $\mathrm{pH} 5.0$ and 7.5 respectively, it showed poor optical contrast of the kidney sections when compared with eosin.

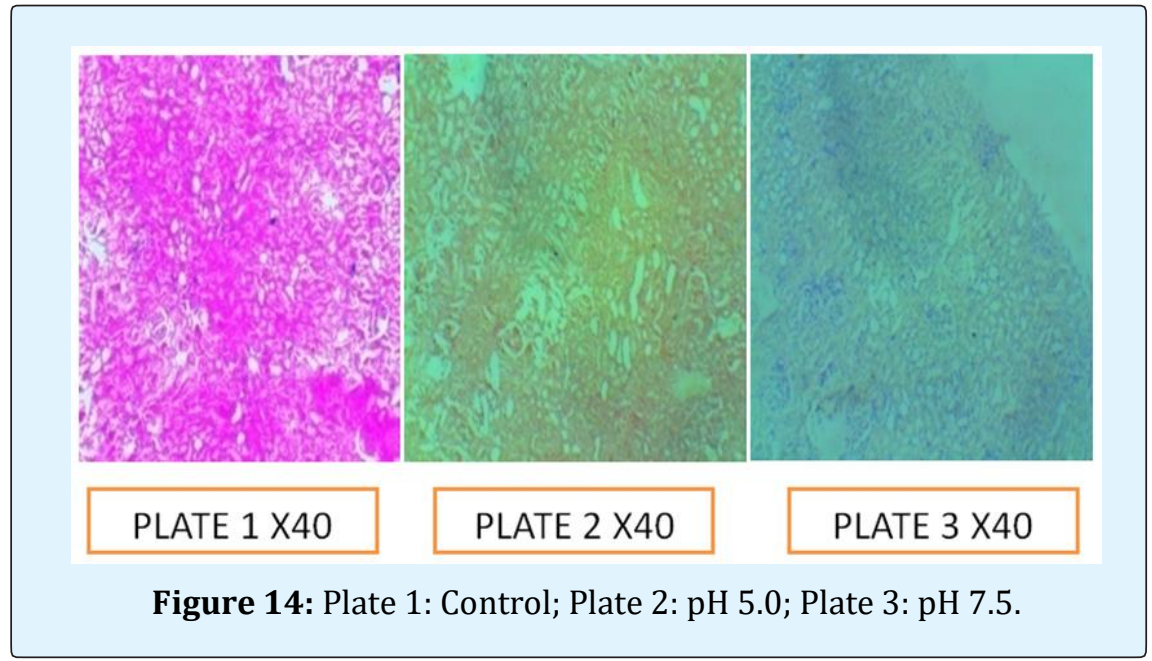

\section{Group P: Interaction of acetone extract of Bixa on Kidney tissue}

The above Group P photomicrographs were the kidney sections of adult Wistar rat, plate 1 is the controlled sections stained with haematoxylin and eosin dye, demonstrated glomerular capsule and podocytes while plate 2 and 3 were the photomicrograph sections of the kidney sections stained with Haematoxylin and counter stained with acetone seeds extracts of bixa orellanna at pH 5.0 and 7.5 respectively, it showed poor optical contrast of the kidney sections when compared with eosin.

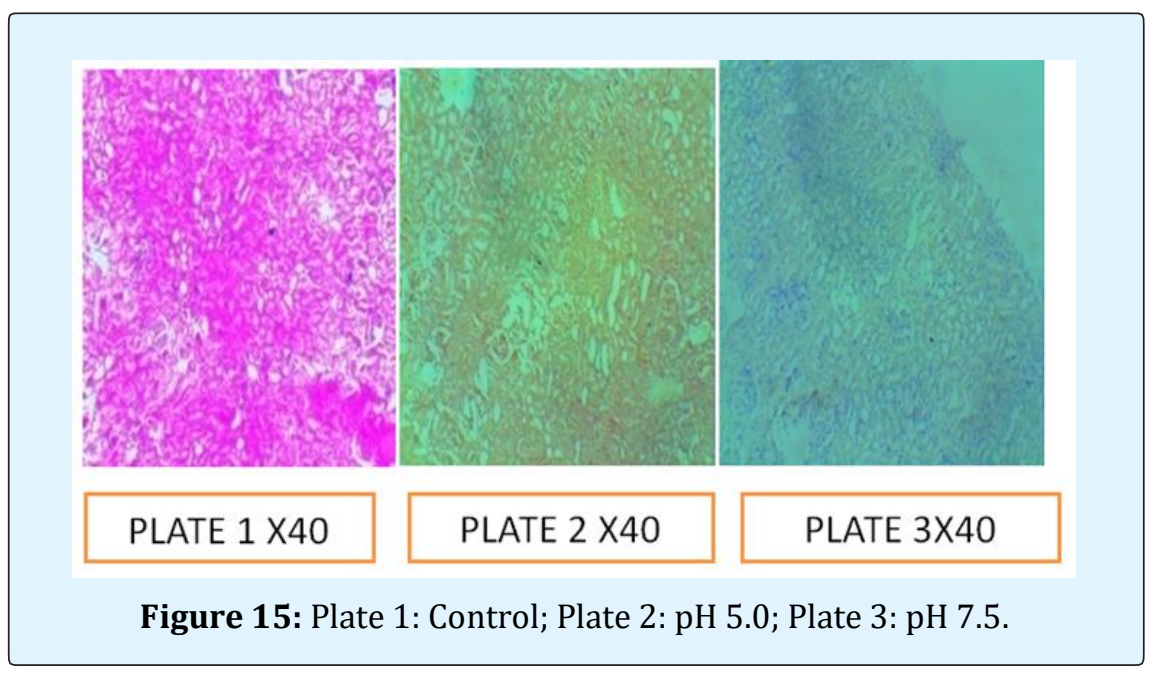

\section{Group Q :Interaction of Column Fraction (9.0- 25) of Bixa Extracts on Liver Tissue}

The aboveGroup Q photomicrograph were the liver sections of adult Wistar rat, plate 1 is the controlled sections stained with haematoxylin and eosin dye, the dye demonstrated the central vein of the liver and portal triad while plate 2 and 3 were the photomicrograph sections of the liver sections stained with Haematoxylin and counter stained with column fraction (9.0-25) of bixa orellanna seeds extractsat $\mathrm{pH} 5.0$ and 7.5 respectively, however, the optical differentiation of the staining interactions were not better than the Eosin control counter stain due to its 
poor nuclear cytoplasmic appearance of the tissue and yellowish background deposition.

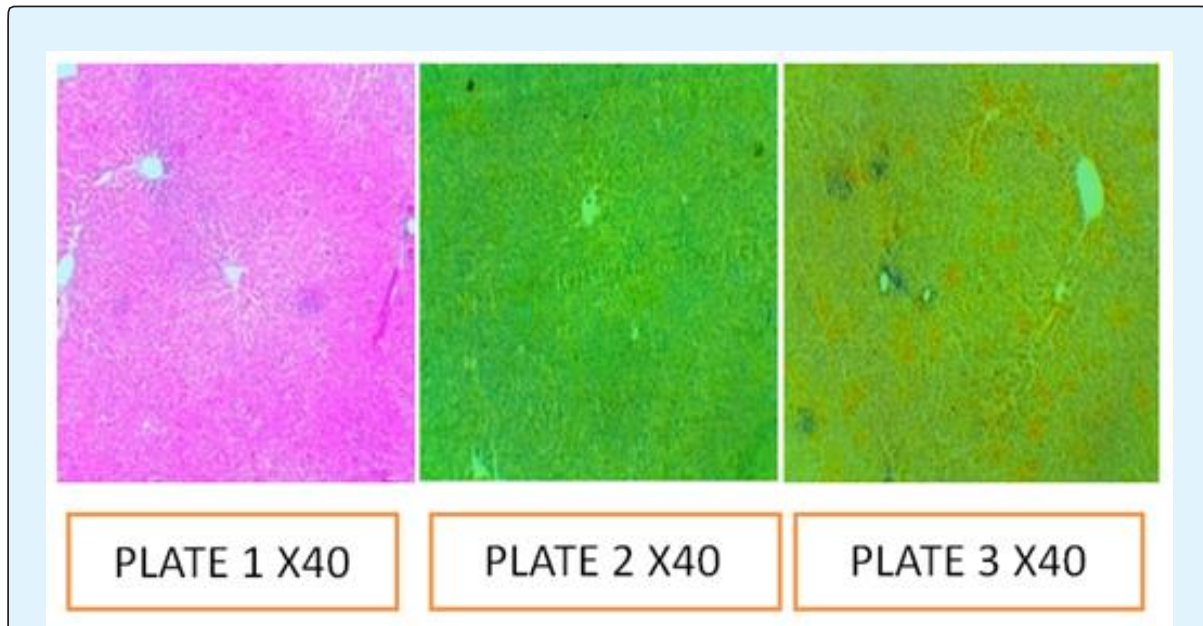

Figure 16: Plate 1: Control; Plate 2: pH 5.0; Plate 3: pH 7.5.

\section{Group R: Interaction of Column Fraction (9.0- 25) of Bixa on Brain Tissues}

The above Group R photomicrographs were the brain sections of adult Wistar rat, plate 1 is the controlled sections stained with haematoxylin and eosin dye, the dye demonstrated the white and gray matter while plate 2 and 3 were the photomicrograph sections of the brain sections stained with Haematoxylin and counter stained with ethanolic seeds extracts of bixa orellanna at $\mathrm{pH} 5.0$ and 7.5 respectively, however, the optical differentiation of the interactions of column fraction (9.0-25) of ethanolic extracts were not better than the Eosin control counter stain due to its poor cytoplasmic appearance of the tissue and yellowish background deposition.

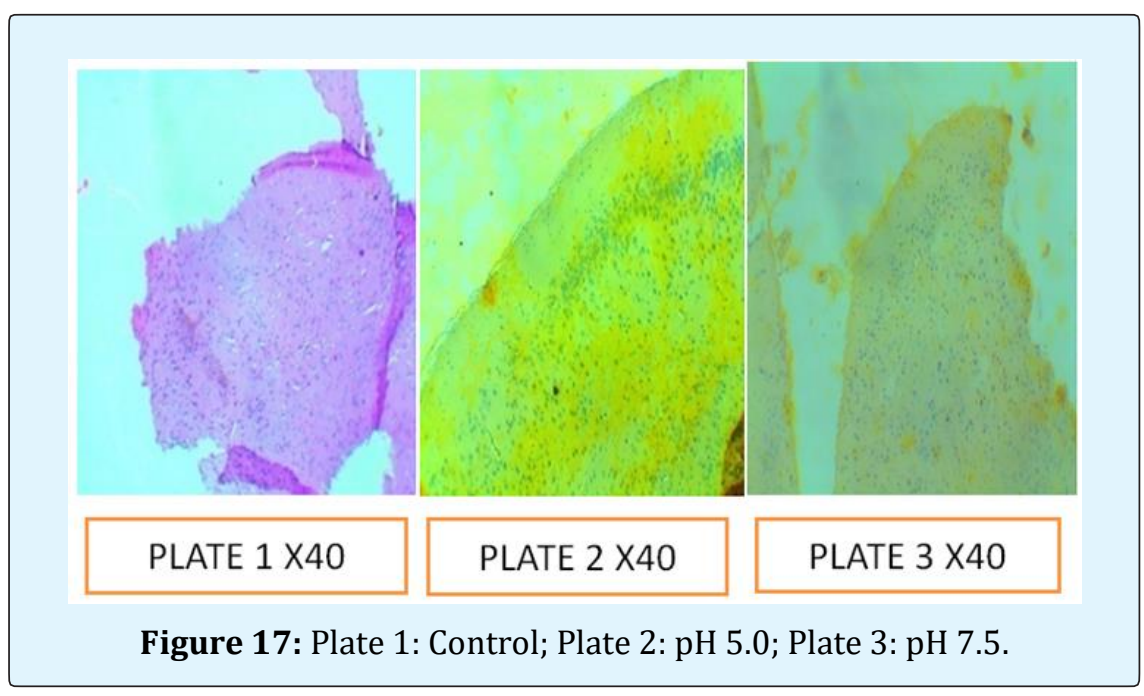

Group S: Interaction of Column Fraction (9.025) of Bixa on Intestine Tissue

The above Group S photomicrographs were the intestine sections of adult Wistar rat, plate 1 is the controlled sections stained with haematoxylin and eosin dye, the dye demonstrated the goblet cells and gastric pit while plate 2 and 3 were the photomicrograph sections of the intestine sections stained with Haematoxylin and counter stained with ethanolic seeds extracts of bixa orellanna at $\mathrm{pH} 5.0$ and 7.5 respectively, however, the 
optical differentiation of the interaction of the column fraction (9.0-25), were not better than the Eosin control counter stain due to its poor cytoplasmic appearance of the tissues and yellowish background deposition.

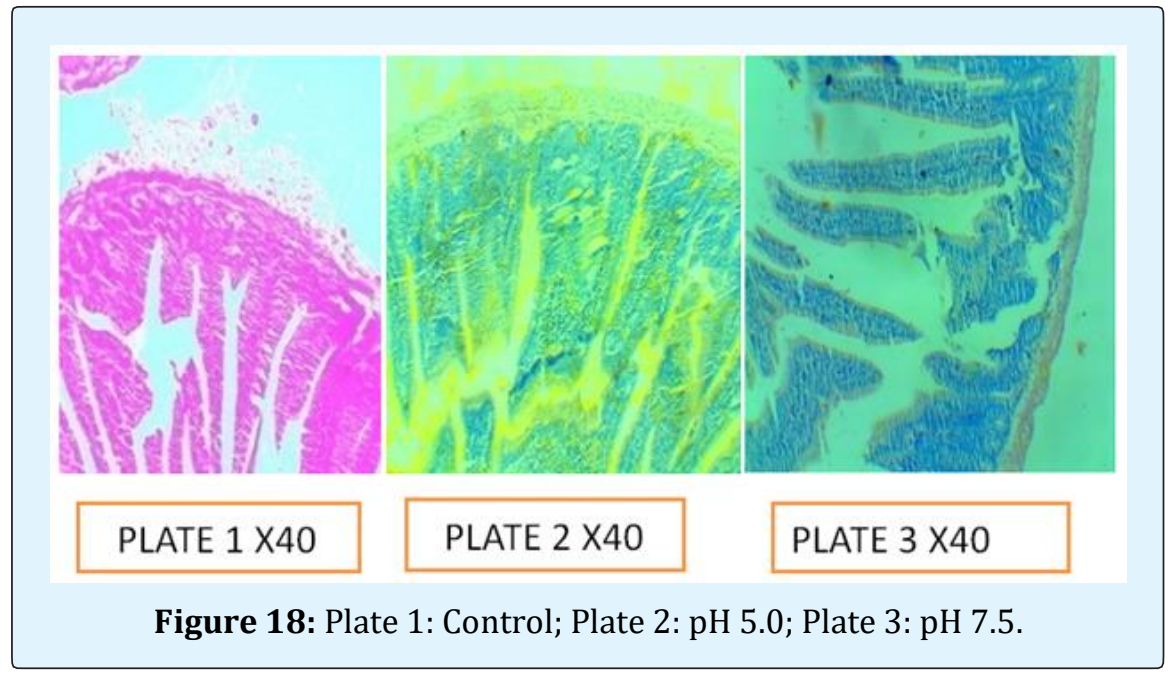

\section{Group T: Interactions of Column Fraction (9.0- 25) of Bixa on Kidney Tissues}

The above Group T photomicrographs were the kidney sections of adult Wistar rat, plate 1 is the controlled sections stained with haematoxylin and eosin dye, the dye demonstrated glomerular capsule and podocytes while plate 2 and 3 were the photomicrographs sections of the kidney sections stained with Haematoxylin and counter stained with column fraction (9.0-25) of bixa orellanna at pH 5.0 and 7.5 respectively, it showed poor optical contrast of the kidney sections when compared with eosin.

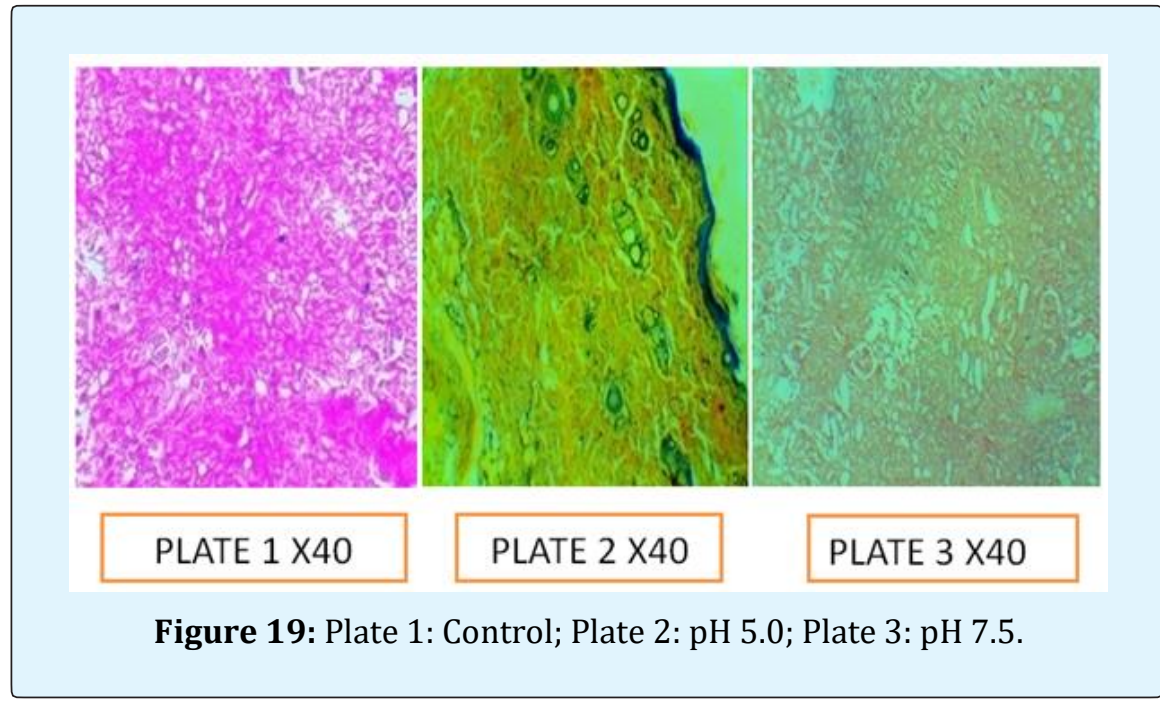

\section{Group U: Interactions of bixa Extract on Blood Smear}

The above Group U photomicrographs were the blood smear of Wistar rat, plate 1 is the controlled slide stained with giemsa dye, the dye demonstrated the morphology of red blood cells, white blood cells and platelets while plate 2 and 3 were the photomicrographs of smear slides stained with column fractions (9.0-25) of bixa orellanna at pH 5.0 and 7.5 respectively, it showed that no staining interaction was observed when compared with giemsa dye. 


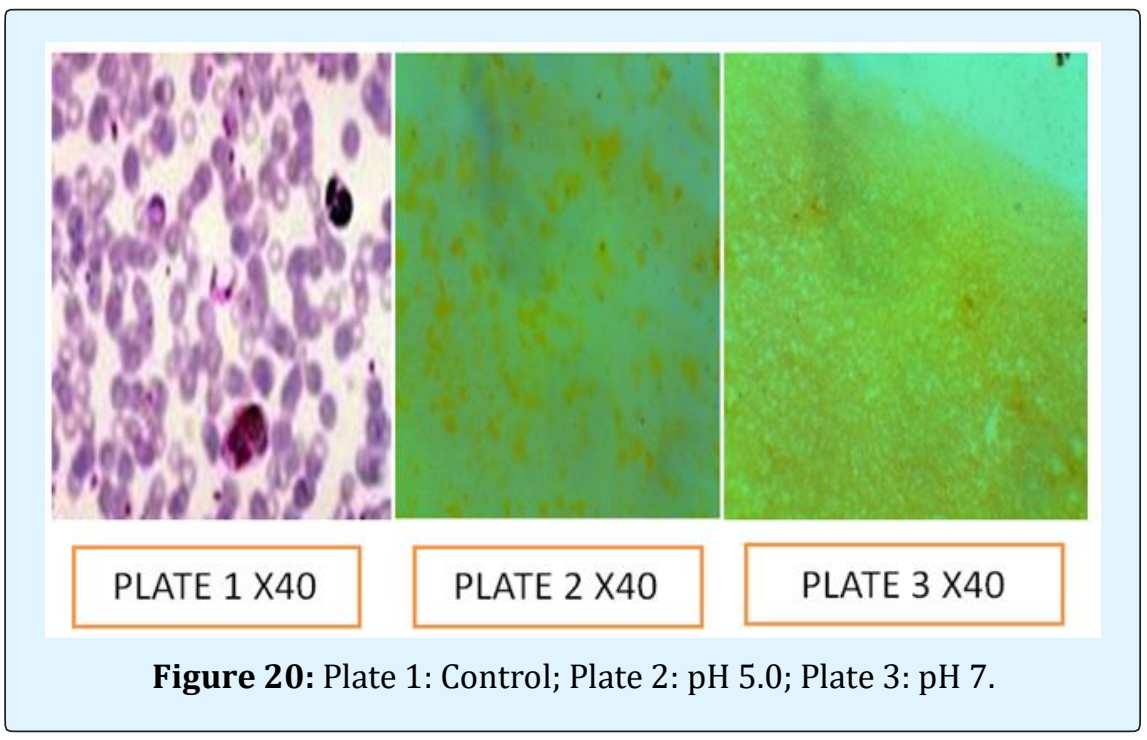

\section{Discussion}

The maceration extraction method was used to extract the Bixa orellana powder using three reagents; Acetone, Ethanol and water with a percentage yield of $30 \%$, $22.97 \%$ and $19.00 \%$ respectively and these comparisons between them shown that bixa orellana seed powder has significant extractive values in different solvents and it is in agreement with results reported by Sangeeta $S$, et al. [7] and James $\mathrm{S}$ [8]. The staining reaction at various $\mathrm{pH}$ shows that Bixa orellana aqueous extract staining reaction is poor and at lesser $\mathrm{pH}$, it alters the histology of the liver and also has yellowish background appearance, in the brain it may not be used as the yellowish background appearance is capable of misleading result when related to jaundice. The dye can also be called a neutral dye or compound dye for reacting at both acidic and basic medium on histology sections of the brain, liver, kidney, skin and intestine as a counter stain to haematoxylin however, there was no significant reaction observed with blood film smear. Though the above reactions at different $\mathrm{pH}$ can be related to chemical theory of staining as explained by Avwioro [9], that certain staining reactions on tissue depends on $\mathrm{pH}$ in relation to the iso-electric points of the dye molecules, best staining interaction was observed with alcohol extract though with less \% yield when compared with acetone extract but acetone extract is better when compared with the staining reaction of water extract. Though no significant reaction was observed in blood smear film and similar results were observed when elution of different collections were compared. It was also discovered that most significant staining interactions occurred within 9.0

- 25.0 fraction elution and it was observed that this fraction has the highest retention factor. Bixa orellana is not a perfect dye to be used for staining brain, liver and placenta tissue sections as it may cause a false positive result in conditions like bilirubin due to its diagnostic importance during autopsy, obstructive jaundice and meconium respectively due to its yellowish background appearance. Bixa orellana extracts can be called a compound dye or neutral dye due to its interaction with tissues on both pH 5.0 and 7.5.

\section{Conclusion}

Bixa orellana seeds extract has a better yield with acetone but ethanol extract has a better optical contrast when compared with acetone and water, Bixa orellana extract cannot be used as a substitute to eosin due to its yellowish background colouration that may cause false positive result. Bixa orellana contains many compounds as was reported in phytochemical screening and Thin Layer Column Chromatography but with a single chromophoric group, Bixa orellana extract is a compound dye since the staining occurred at both $5.0 \mathrm{pH}$ and 7.5 on the tested tissue but cannot be used to stain blood smear since no staining reaction was observed with the extract however, giemsa stain is a polychromatic dye which stains red blood cells, white blood cells and platelets in varying colours.

\section{Ethical Approval}

All authors hereby declare that all experiments have been examined and approved by the appropriate ethics research committee and have therefore been performed 
in accordance with ethical standard laid down in the 1964; declaration of Helsinki

\section{Conflicts of Interest}

All authors have declared that there is no conflict of interest.

\section{References}

1. Joly AB (1993) Botanica: Introducao a taxonomia vegetal, 11 ${ }^{\text {th }}$ (Edn.), Nacional, Sao Paulo, Brazil 38: 779-784.

2. Ribeiro JA, Oliveira DT, Passos ML, Barazzo MAS (2005) The use of nonlinearity measures to discriminate the equilibrium moisture equations for Bixa orellana seeds. Journal of Food Engeneering 66(1): 63-68.

3. Akshatha V, Giridhar P (2012) Bacterial growth inhibition potential of annatto plant parts. Asian Pacific Journal of Tropical Biomedica 2(3): S1879S1872.

4. Nguyen PM (2014) Different factors influencing to annatto dye extraction in Bixa orelana L. seeds.
International Journal of Multidisciplinary Research and Development 1(5): 81-84.

5. Dolores JY, María TM, Ivan LV, Antonio FB, Sofia Vicente-Palomino, et al. (2008) Arché. Publicación del instituto universitario de restauración del patrimonio de la upv-Núm. Journal of the heritage conservation institute of the Polytecnical University of Valencia 3: 154-158.

6. Silva GF, Gamarra FMC, Oliveira AL, Cabral FA (2008) Extraction of bixin from annatto seeds using supercritical carbon dioxide. British Journal of Chemical Engineeing 25(2): 419-426.

7. Sangeeta S, Manasi M, Chetna S, Suresh K, Murthy SN (2015) Comparative phytochemical screening of qualitative and quantitative parameters of bixa orellana l. World Journal of Pharmacy And Pharmaceutical Sciences 4(12): 1001-1017.

8. James S (2006) Annatto Extracts (PDF). Chemical and Technical Assessment Manual. JECFA.

9. Avwioro (2010) Theory of staining. Histochemistry and Tissue pathology, Principles and Techniques Textbook, $3^{\text {rd }}$ (Edn.), Claverianum Press, pp: 142-145. 